\title{
Lidil
}

Revue de linguistique et de didactique des langues

$60 \mid 2019$

Langues des signes et genres discursifs

Stéphane Bikialo et Julien Rault (dir.), Imaginaires de la ponctuation. Ordre et inquiétude du discours, revue $\operatorname{Lin} x, \mathrm{n}^{\circ} 75$

Presses universitaires de Paris Nanterre, 2017, 224 p.

Luca Pallanti

CpenEdition

Journals

Édition électronique

URL : http://journals.openedition.org/lidil/6649

DOI : $10.4000 /$ lidil.6649

ISSN : $1960-6052$

Éditeur

UGA Éditions/Université Grenoble Alpes

Édition imprimée

ISBN : 978-2-37747-164-5

ISSN : $1146-6480$

Référence électronique

Luca Pallanti, « Stéphane Bikialo et Julien Rault (dir.), Imaginaires de la ponctuation. Ordre et inquiétude du discours, revue Linx, no 75 », Lidil [En ligne], 60 | 2019, mis en ligne le 01 novembre 2019, consulté le 25 septembre 2020. URL : http://journals.openedition.org/lidil/6649; DOI : https://doi.org/10.4000/ lidil.6649

Ce document a été généré automatiquement le 25 septembre 2020.

(C) Lidil 


\section{Stéphane Bikialo et Julien Rault (dir.), Imaginaires de la ponctuation. Ordre et inquiétude du discours, revue Linx, $\mathrm{n}^{\circ} 75$}

Presses universitaires de Paris Nanterre, 2017, 224 p.

Luca Pallanti

\section{RÉFÉRENCE}

Stéphane Bikialo et Julien Rault (dir.), Imaginaires de la ponctuation. Ordre et inquiétude du discours, revue Linx, $\mathrm{n}^{\circ}$ 75, Presses universitaires de Paris Nanterre, 2017, $224 \mathrm{p}$.

1 L'idée sous-jacente qui traverse le $\mathrm{n}^{\circ} 75$ de la revue Linx, dirigé par S. Bikialo et J. Raoult, coïncide avec la volonté d'explorer les représentations liées à la ponctuation au fil des siècles. Si pendant longtemps la ponctuation n'a pas été considérée comme un objet d'étude linguistique à part entière, à partir des années 1980 le regard des linguistes s'est finalement posé sur ce domaine mystérieux. Les auteurs interrogent ainsi la dimension perceptive relative aux signes de ponctuation, pour aboutir à « une représentation en forme d'imaginaire de la ponctuation et des signes qui la composent» (p.10). L'originalité de l'ouvrage est constituée par l'alléchante association entre les sentiments de la ponctuation et l'analyse des pratiques de publics hétérogènes.

2 Les nombreuses problématiques évoquées par les onze articles sélectionnés questionnent les aspects relatifs à la portée structurante des signes de ponctuation. Il s'agit d'explorer les liens qui se créent entre pratiques ponctuantes et syntaxe, le rôle de la prosodie, les implications socio-idéologiques de certains signes, les relations entre l'univers musical et l'univers linguistique, et les effets stylistiques qui en dérivent. De la 
Renaissance à nos jours, les différentes études se concentrent tantôt sur l'étude des textes fondateurs, tantôt sur l'analyse des discours épilinguistiques et des corpus écrits.

C'est dans une perspective historico-stylistique que l'on peut situer les études de C. Pagani-Naudet, G. Stouffi, J. Raoult et J. Dürrenmatt. Dans cette première partie de l'ouvrage, on peut apprécier l'effort de reconstruction de l'imaginaire de la ponctuation $\mathrm{du} \mathrm{XVI} \mathrm{I}^{\mathrm{e}}$ au $\mathrm{XIX}^{\mathrm{e}}$ siècle. Ainsi, la première étude est consacrée à la vision phonocentriste de Louis Meigret dans le Tretté de la grammere françoeze (1550) et aux points de différence avec le grammairien Dolet. L'idée défendue est celle d'une complémentarité entre prosodie et signe graphique, où Meigret redessine le vocabulaire de la ponctuation tout en introduisant la fonction structurante des signes sur la syntaxe. Cette réflexion se poursuit dans l'article de G. Stouffi où l'on aborde la dimension idéale, voire idéalisée, des signes de ponctuation des grammaires latines jusqu'à la grammaire de Port-Royal. Les allers-retours entre l'interprétation subjective des signes et leur caractérisation objective culminent avec une réflexion sur les pratiques ponctuantes au Xix ${ }^{e}$ siècle, âge d'or des philologues, qui «va développer l'activité de ponctuation des textes du passé, exerçant de la sorte un "magistère" dans le domaine de la ponctuation » (p. 47).

Ponctuer rime parfois avec séduire. Ainsi, J. Raoult montre de manière suggestive les émotions et la sensualité des points de suspension au XvIII ${ }^{\mathrm{e}}$ siècle, où la pensée libertine utilisait la ponctuation comme un moyen de transgression, suivant la tradition subversive des œuvres satiriques du passé. Les implications stylistiques des signes de ponctuation sont multiples et dans son étude sur la coupure, J. Dürrenmatt cherche à montrer à travers l'analyse de différents passages littéraires du XIX ${ }^{\mathrm{e}}$ siècle les effets de style de la phrase hachée, sa dimension linguistique et sa portée référentielle. Ces recherches sont complétées par une analyse comparative de la pratique du tiret, ce qui a le mérite de mettre en avant la complexité de la portée énonciative de ce signe et les problématiques interprétatives qui en découlent (C. Rannoux).

Néanmoins, une description de l'imaginaire de la ponctuation ne peut pas faire abstraction des discours épilinguistiques qui la caractérisent. Dans la deuxième partie de l'ouvrage, S. Bikialo expose les résultats d'une enquête réalisée auprès de 40 auteurs contemporains sur leur vision de la ponctuation et sur leurs pratiques ponctuantes. Il émerge un imaginaire de la ponctuation spontanément normatif, essentiellement inchangé pendant 40 ans, où « la norme est plutôt de l'ordre de ce qui ne se voit pas, dont la matérialité n'est plus sensible voire esquivée» (p.116). Cette invisibilité de la norme est source d'inquiétude et participe de la prégnance de la notion de valeur expressive chez É. Bordas. L'auteur invite le lecteur à regarder de près la frontière entre expression individualisante et expression généralisante commune, lieu où les signes de ponctuation semblent échapper au volet déterministe des grammaires.

Un imaginaire de la ponctuation peut être également envisagé à partir de l'analyse de corpus de textes plus étendus. C'est notamment le cas des études de A. Steuckardt et de A.-L. Kiviniemi. Dans le premier cas, où l'on explore les échanges épistolaires des peulettrés au sein du «Corpus 14 », on peut retenir l'existence d'un continuum avec le corpus des peu-lettrés de la Révolution; le trait d'union serait la rareté des signes de ponctuation et l'usage globalement normatif des signes. Dans le deuxième cas, on relève à quel point la ponctuation peut être considérée comme une marque de la subjectivité du scripteur. Une troisième étude sur l'emploi de la virgule dans un corpus de productions écrites d'apprenants brésiliens met en évidence l'écart existant entre 
les conventions syntaxiques et la présence de l'oralité dans l'activité de ponctuation des scripteurs (G. Soncin et L. Tenani).

7 Somme toute, c'est bien la tension entre une conception théorique, normative, de la ponctuation et le rapport à l'oralité, plus affectif et sensible, qui semble bien caractériser l'imaginaire de la ponctuation. Cet objet aux contours flous a subi plusieurs tentatives de reformes durant les siècles (M. Ponge) et des propositions innovantes, parfois provocantes, ont vu le jour. Des critiques rousseauiennes aux provocations de Paul Valéry, du modèle de l'écriture musicale aux idéogrammes de ponctuation, l'univers des pratiques ponctuantes ne cesse de charmer et de questionner les spécialistes de la langue de tout horizon de par sa substance à la fois formelle et sensorielle.

\section{AUTEURS}

LUCA PALLANTI

LIDILEM (EA 609), Université Grenoble Alpes 